\title{
Automated quantification and analysis of mandibular asymmetry
}

Darvann, T. A.; Hermann, N. V.; Larsen, P.; Ólafsdóttir, Hildur; Hansen, I. V.; Hove, H. D.; Christensen, L.; Rueckert, D.; Kreiborg, S.

Published in:

2010 IEEE International Symposium on Biomedical Imaging: From Nano to Macro

Link to article, DOI:

$10.1109 /$ ISBI.2010.5490320

Publication date:

2010

Document Version

Publisher's PDF, also known as Version of record

Link back to DTU Orbit

Citation (APA):

Darvann, T. A., Hermann, N. V., Larsen, P., Ólafsdóttir, H., Hansen, I. V., Hove, H. D., Christensen, L. Rueckert, D., \& Kreiborg, S. (2010). Automated quantification and analysis of mandibular asymmetry. İ 2010 IEEE International Symposium on Biomedical Imaging: From Nano to Macro (pp. 416-419). IEEE. https://doi.org/10.1109/ISBI.2010.5490320

\section{General rights}

Copyright and moral rights for the publications made accessible in the public portal are retained by the authors and/or other copyright owners and it is a condition of accessing publications that users recognise and abide by the legal requirements associated with these rights.

- Users may download and print one copy of any publication from the public portal for the purpose of private study or research.

- You may not further distribute the material or use it for any profit-making activity or commercial gain

- You may freely distribute the URL identifying the publication in the public portal 


\title{
AUTOMATED QUANTIFICATION AND ANALYSIS OF MANDIBULAR ASYMMETRY
}

\author{
Tron A. Darvann ${ }^{1}$, Nuno V. Hermann ${ }^{1,2}$, Per Larsen ${ }^{1}$, Hildur Ólafsdóttir ${ }^{3}$, Izabella V. Hansen ${ }^{4}$, \\ Hanne D. Hove ${ }^{5}$, Leif Christensen ${ }^{6}$, Daniel Rueckert ${ }^{7}$, Sven Kreiborg ${ }^{1,2,5}$
}

1) 3D Craniofacial Image Research Laboratory (Copenhagen University Hospital Rigshospitalet; School of Dentistry, University of Copenhagen; and DTU Informatics, Technical University of Denmark), Copenhagen, Denmark

2) Pediatric Dentistry and Clinical Genetics, School of Dentistry, University of Copenhagen, Copenhagen, Denmark

3) DTU Informatics, Technical University of Denmark, Lyngby, Denmark

4) Orthodontics, School of Dentistry, University of Copenhagen, Copenhagen, Denmark

5) Department of Clinical Genetics, Copenhagen University Hospital Rigshospitalet, Copenhagen, Denmark

6) Department of Neurosurgery, Århus University Hospital, Århus, Denmark

7) Visual Information Processing, Department of Computing, Imperial College London, London, UK

\begin{abstract}
We present an automated method of spatially detailed 3D asymmetry quantification in mandibles extracted from CT and apply it to a population of infants with unilateral coronal synostosis (UCS). An atlas-based method employing nonrigid registration of surfaces is used for determining deformation fields, thereby establishing detailed anatomical point correspondence between subjects as well as between points on the left and right side of the mid-sagittal plane (MSP). Asymmetry is defined in terms of the vector between a point and the corresponding anatomical point on the opposite side of the MSP after mirroring the mandible across the MSP. A principal components analysis of asymmetry characterizes the major types of asymmetry in the population, and successfully separates the asymmetric UCS mandibles from a number of less asymmetric mandibles from a control population.
\end{abstract}

Index Terms - Asymmetry, non-rigid registration, atlas-based methods, craniofacial malformations, unilateral coronal synostosis

\section{INTRODUCTION}

Cranial and facial asymmetry is a characteristic of many congenital and acquired craniofacial malformations, and determination of amount, localization and spatial extent of asymmetry is important in the contexts of differential diagnostics as well as evaluation of disease and treatment progression. In some craniofacial malformations, the mandible presents with an asymmetry that needs to be quantified and, sometimes, surgically corrected. In individuals with unilateral coronal synostosis (UCS) one of the coronal sutures is prematurely fused, leading to a compensatory asymmetric growth of the skull that also affects the mandible. UCS asymmetry has previously been assessed using methods based on manual point landmarking, e.g. in [1]. Recently, we have developed methods for asymmetry quantification that provide spatially dense asymmetry values across soft tissue head and face surfaces obtained in surface scanners [2,3]. These methods are based on non-rigid registration of a fully symmetric (head or face) template to each individual, thereby transferring the knowledge of left-right anatomical correspondence in the template to the individuals. The non-rigid registration is based on manual point-landmarking and thin-plate-spline (TPS) deformation followed by closest point deformation (CPD), inspired by [4]. These methods often fail, however, when applied to the more complex anatomy of the mandible due to incorrect matches by the simple CPD. The problems are avoided using free-form deformation methods [5]. We have previously developed such computational atlas-based methods that automatically determine deformation fields in images of craniofacial anatomy [6,7] and used these for asymmetry quantification [8]. The methods have been applied with success to images of anatomy including the UCS mandible. In the present study we apply and validate a surface based version of the non-rigid registration method [9]. The present study contributes three novel additions to the methodology: 1) Automated determination of point correspondences for the mandible by use of B-spline based non-rigid surface registration, 2) Automated rigid registration to an anatomical coordinate system, and 3) Scale-invariant definition of asymmetry. The method is applied to UCS mandibles with varying amount of asymmetry, as well as to a control population with Apert syndrome which have unaffected (nearly normal) mandibles. 


\section{MATERIAL}

The material consisted of polygonal surface models of mandibles extracted from pre-operative CT scans of 14 infants with UCS (mean age 7 months, range 4-16 months) and 5 infants with Apert syndrome (mean age 6 months, range 2-9 months). CT scans had been obtained for treatment purposes at the University Hospitals of Copenhagen and Århus, respectively.

\section{METHODS}

Before application of the method, CT data had been oriented according to a standard head orientation using the midsagittal plane (MSP) and the Frankfort horizontal plane (see e.g. [1]). Furthermore, the mandibles had been segmented in the CT image data and surface representations created. For validation purposes, 14 of the mandibles were manually landmarked with 30 landmarks by two different observers. Mandibles of subjects with UCS had been mirrored, when necessary, in order to have the dysmorphology on the same (right) side.

The method consisted of the following eight main steps:

1) Creation of an initial symmetric template mandible. One of the mandibles were selected and deformed to an average landmark configuration by use of TPS. A symmetric template mandible was created by cutting the resulting mandible at the midline (symphysis), discarding one side, mirroring the other side, and finally stitching the mirrored and original sides together.

2) Alignment of each individual mandible to the template. This was carried out by translating the center-of-gravity $(\operatorname{cog})$ of the individual to the $\operatorname{cog}$ of the template.

3) Creation of an average symmetric atlas mandible.

For each of the mandibles, the symmetric template was deformed to the individual mandible by B-spline based nonrigid surface registration minimizing the squared distances between the surfaces [10], implemented in the Image Registration Toolkit (www.doc.ic.ac.uk/dr/software/). The deformed template was kept as a representation of the individual mandible for the further analysis. In this way, all mandibles inherited the polygonal mesh connectivity of the template, and a population mean mandible could be created by simple point-wise averaging.

\section{4) Bias reduction.}

In order to reduce bias [4] towards the shape of the single mandible used for the template creation, steps 2 and 3 were repeated with the symmetric template replaced by the average symmetric atlas created in step 3 .
5) Landmarking and segmentation of the atlas.

The atlas was landmarked with 30 anatomical point landmarks, and the alveolus (tooth region) was segmented.

6) Alignment according to anatomical coordinate system.

To facilitate interpretation of calculated asymmetry, the normal (left) side of the mandible (in subjects with UCS) was rigidly registered to the left side of the atlas mandible using the iterated closest point (ICP) algorithm. Prior to ICP transformation, some of the landmarks placed in the atlas (in step 5) were automatically transferred to the individual mandible and used for initial alignment as well as for anisotropic scaling of the template to each individual mandible. The scaling was seen to be important for the ICP to provide plausible registrations.

\section{7) Quantification of asymmetry in each individual.}

The asymmetry at a point $P$ on the left side of the MSP was determined in terms of the 3D vector A (Figure 1).

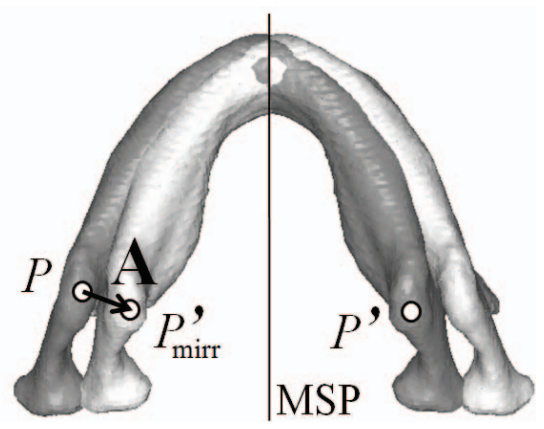

Fig. 1. Schematic illustration of asymmetry quantification in an asymmetric mandible (the mandible with dark gray shading in the figure). The asymmetry vector $\mathbf{A}$ is defined as the vector between the points $P$ and $P^{\prime}{ }_{\text {mirr }} P$ marks a point on one side of the mandible, $P^{\prime}$ marks the anatomically corresponding point on the other side. The light shaded mandible is the same mandible after it has been mirrored across the mid-sagittal plane. $P^{\prime}$ mirr marks the location of $P$ ' after the mirroring.

Formally, the amount (or magnitude) of asymmetry was defined as $A_{m}=\|\mathbf{A}\| \cdot f$, where \|\| denotes the Euclidian norm and $f=S_{i} / S_{\text {atlas }}$ is a scale factor correcting for differences in mandible size among individuals; $S_{i}$ and $S_{\text {atlas }}$ being the centroid size of an individual mandible and the atlas mandible, respectively. The scale factor $f$ was introduced as it may be observed that the vector A scales proportionately with mandibular size. Intuitively, asymmetry should be identical in two different mandibles having the same shape but differing size (e.g. a child's and an adult's mandible, respectively). $f$ provides a normalization such that the asymmetry becomes invariant to size. The three Cartesian vector components of $\mathbf{A}$ provide the amount of asymmetry in the transverse $(t)$, vertical $(v)$ and sagittal $(s)$ directions, respectively: $A_{t}=\operatorname{comp}_{x} \mathbf{A} \cdot f ; A_{v}=\operatorname{comp}_{y} \mathbf{A} \cdot f$; $A_{s}=\operatorname{comp}_{z} \mathbf{A} \cdot f$. 
8) Construction of statistical models of asymmetry.

Principal components analysis as in [2] was applied to the asymmetry vectors in each of the three directions, respectively, providing the major modes of variation in the population under study. The region containing teeth, segmented in step 5, was excluded from the analysis due to the variable amount of tooth eruption between individuals. Also, a mean mandible was created for the population, as well as for the two sub-populations (UCS and Apert syndrome, respectively).

\section{RESULTS}

The non-rigid registration was validated in two ways.

1) The ability of the atlas to achieve the shape of the individual mandible was determined by, for each point in the deformed template, calculating the closest point distance to the original individual shape, and finally averaging over all individuals (Figure 2). The spatially averaged mean error was found to be $0.05 \mathrm{~mm}$. Mean errors of up to $0.7 \mathrm{~mm}$ occurred in the alveolar area containing teeth.

2) The use of closest point distances for validation did not necessarily reveal the ability of the method to match in the anatomical sense. Therefore, a landmark based validation was carried out, comparing the automatically determined landmark locations with manually placed landmarks in each individual mandible. The result is depicted in Figure 3 alongside an inter-observer landmarking error, for comparison. RMS error varied from less than $1 \mathrm{~mm}$ to $5 \mathrm{~mm}$ depending on the landmark, and, on average, the error was 1.7 times larger than the inter-observer error.

Mean shapes for the UCS and Apert populations, respectively, color coded according to mean asymmetry of the respective groups, are shown in Figure 4.

Figure 5 shows the first two PCA modes for transverse and vertical asymmetry, respectively, in models containing UCS individuals alone. For the transverse asymmetry, the first two modes captured 71 and $22 \%$ of the variation in the data, corresponding to a deviation of the ramus towards the MSP and a variation of curvature along the entire length of the mandible, respectively. For the vertical asymmetry, the first two modes captured 94 and $3 \%$ of the variation, corresponding to a vertical bending/displacement of the whole mandible and of the condyle, respectively.

Figure 6 shows a plot of PC 1 for the vertical asymmetry versus PC 1 for the transverse asymmetry in a model containing mandibles from both UCS and Apert syndrome individuals, and demonstrates the ability of the model to discriminate between the two populations based on asymmetry.

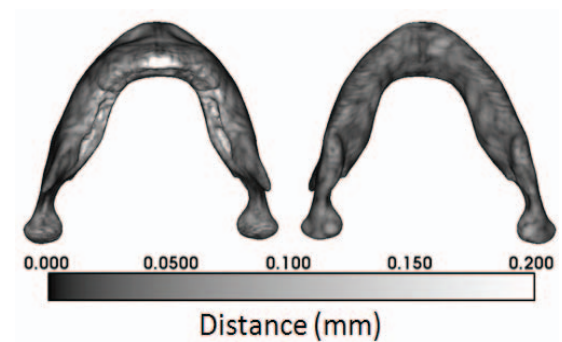

Fig. 2. Mean closest point distance between estimated and true shapes, color coded onto mean UCS shapes seen in top (left) and bottom (right) views.

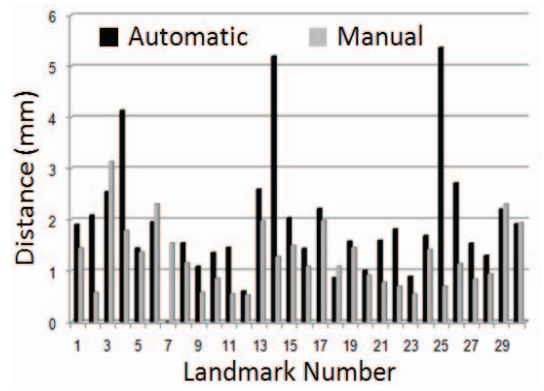

Fig. 3. Landmark based validation. RMS error of non-rigid registration (black columns) is shown next to inter- observer RMS error (gray columns) for 30 landmarks.

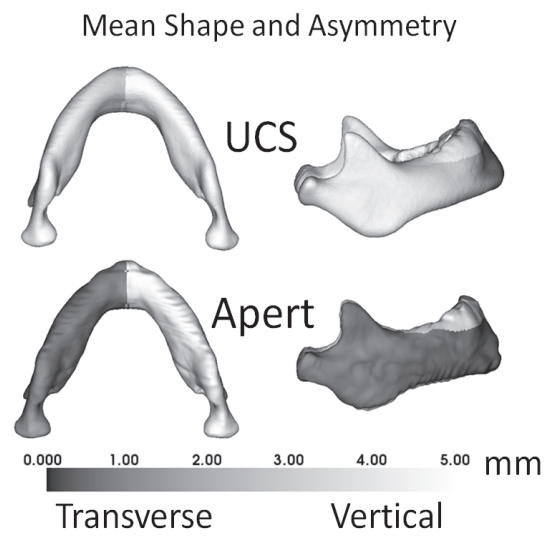

Fig. 4. Mean asymmetry for the UCS and Apert populations, color coded onto the respective mean shapes, seen in bottom and lateral views. Normal (left) side of the mandible has by definition no asymmetry and is shown in white.

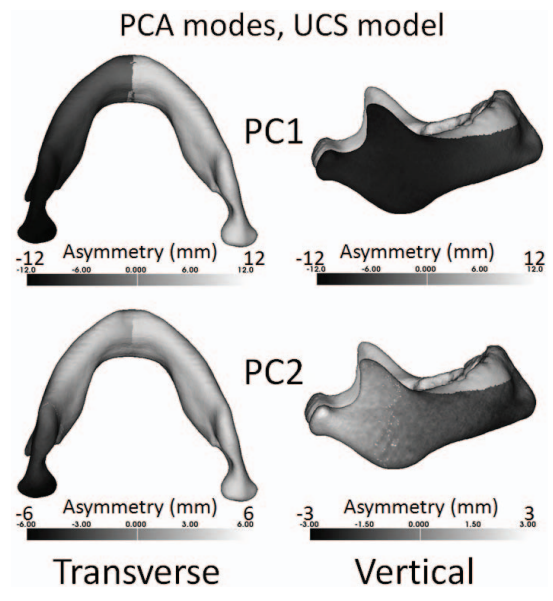

Fig. 5. PCA modes in a model of asymmetry containing UCS mandibles. Colors depict a deviation of 3 standard deviations in $\mathrm{mm}$ from the mean asymmetry. Black and white indicate deviations in opposite directions in the directions as indicated. 


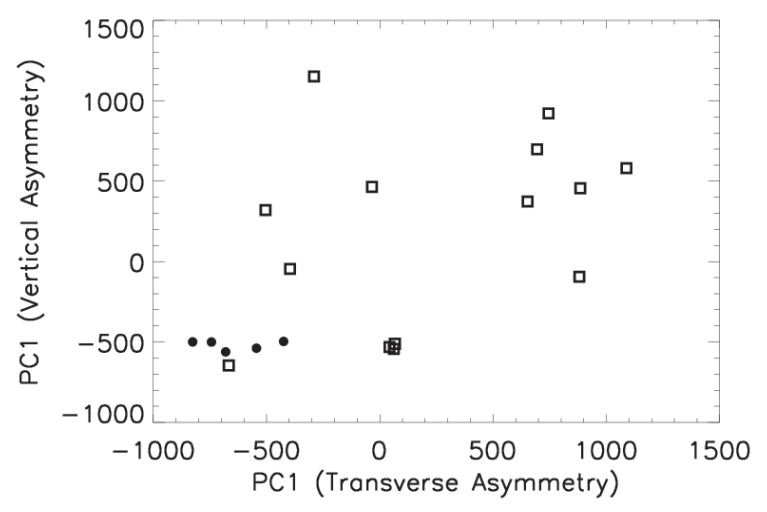

Fig. 6. PCA score plot showing PC1 of vertical asymmetry versus $\mathrm{PC} 1$ of transverse asymmetry. Squares and filled circles represent UCS and Apert individuals, respectively. Least amount of asymmetry is found in subjects located in the lower left corner of the diagram.

\section{DISCUSSION AND CONCLUSION}

The validation results demonstrated that the non-rigid surface registration performed robustly and with sufficient accuracy for quantification of mandibular shape and asymmetry. The method was able to provide detailed asymmetry maps for individual mandibles that correlated well with visual assessment of asymmetry. Significant differences in mean shape and asymmetry between UCS and Apert syndrome mandibles were demonstrated, although in a limited sample of subjects.

Since the malformation in UCS predominantly affects one side of the mandible, the use of an anatomical coordinate system, where asymmetry on the normal side is minimized, offers an alternative and perhaps more intuitively interpretable solution compared to the mathematical coordinate system used in e.g. [2,8].

Statistical analysis of the asymmetry by PCA revealed intuitively interpretable modes of variation, and correctly classified the UCS and Apert mandibles as high- and lowasymmetry mandibles, respectively. The scale-invariance of the asymmetry definition made it possible to compare the mandibular asymmetry of individuals across a range of ages, and was thus a prerequisite for the construction of an interpretable statistical model of asymmetry.

Applications of mandibular asymmetry assessment could be found in areas such as maxillofacial surgery, clinical genetics and anatomy.

\section{Acknowledgements}

For all non-rigid surface registrations, the Image Registration Toolkit (http://www.doc.ic.ac.uk/dr/software/) was used under License from Ixico Ltd. For all surface visualizations, landmarking, ICP transformations and closest point calculations, the VTK-based software landmarker [10] was used (http://www.lab3d.odont.ku.dk /landmarker)

\section{REFERENCES}

[1] Keller M, Hermann NV, Darvann TA, Larsen P, Hove H, Christensen L, Schwartz M, Marsh J, Kreiborg S. Craniofacial morphology in Muenke syndrome. J Craniofac Surg. 2007;18:37486.

[2] Lanche S, Darvann TA, Ólafsdóttir H, Hermann NV, Van Pelt AE, Govier D, Tenenbaum MJ, Naidoo S, Larsen P, Kreiborg S, Larsen R, Kane AA. A statistical model of head asymmetry in infants with deformational plagiocephaly. In: Ersbøll BK, Pedersen KS (Eds.): Image Analysis. Springer Verlag, Berlin. Lecture Notes in Computer Science 2007;4522:898-907.

[3] Darvann TA, Ólafsdóttir H, Hermann NV, Larsen P, Lanche S, Larsen R, Ersbøll BK, Govier D, Van Pelt AE, Kane AA, Hansen IV, Kreiborg S. On the measurement of craniofacial asymmetry. In: Takada K, Kreiborg S, editors. In Silico Dentistry - The Evolution of Computational Oral Health Science. Osaka, Japan: Medigit; 2008. p. 37-41.

[4] Hutton T, Buxton BF, Hammond P, Potts HW. Estimating average growth trajectories in shape-space using kernel smoothing. IEEE Trans Med Imaging 2003;22:747-53.

[5] Rueckert D, Frangi AF, Schnabel JA. Automatic construction of 3D statistical deformation models of the brain using nonrigid registration. IEEE Trans. on Medical Imaging. 2003;22:1014-25.

[6] Ólafsdóttir H, Darvann TA, Hermann NV, Oubel E, Ersbøll BK, Frangi AF, Larsen P, Perlyn CA, Morriss-Kay GM, Kreiborg S. Computational mouse atlases and their application to automatic assessment of craniofacial dysmorphology caused by the Crouzon mutation Fgfr2(C342Y). J Anat. 2007;211:37-52.

[7] Ólafsdóttir H, Lanche S, Darvann TA, Hermann NV, Larsen R, Ersbøll BK, Oubel E, Frangi AF, Larsen P, Perlyn CA, MorrissKay GM, Kreiborg S. A point-wise quantification of asymmetry using deformation fields: application to the study of the Crouzon mouse model. Med Image Comput Comput Assist Interv 2007; p. $452-9$.

[8] Ólafsdóttir H. Analysis of Craniofacial Images using Computational Atlases and Deformation Fields. PhD Thesis, Technical University of Denmark, 2007.

[9] Szeliski R, Lavallée S. Matching 3-D anatomical surfaces with non-rigid deformations using octree-splines. Int J Computer Vision 1996;18:171-86.

[10] Darvann TA. Landmarker: A VTK-based tool for landmarking of polygonal surfaces. In: Takada K, Kreiborg S, editors. In Silico Dentistry - The Evolution of Computational Oral Health Science. Osaka, Japan: Medigit; 2008. p. 160-2. 